\title{
Editorial
}

\section{Diabetes Mellitus and Its Cardiovascular Complications: New Insights into an Old Disease}

\author{
Celestino Sardu $\left(\mathbb{D},{ }^{1}\right.$ Claudio De Lucia $\mathbb{D},{ }^{2}$ Markus Wallner, ${ }^{3}$ and Gaetano Santulli $\mathbb{D}^{4,5}$ \\ ${ }^{1}$ University of Campania "Luigi Vanvitelli", Naples, Italy \\ ${ }^{2}$ Center for Translational Medicine, Lewis Katz School of Medicine, Temple University, Philadelphia, PA, USA \\ ${ }^{3}$ Medical University of Graz, Graz, Austria \\ ${ }^{4}$ Albert Einstein College of Medicine, The "Norman Fleischer" Institute for Diabetes and Metabolism, The "Wilf Family" \\ Cardiovascular Research Institute, New York, NY, USA \\ ${ }^{5}$ University of Naples "Federico II", Naples, Italy
}

Correspondence should be addressed to Celestino Sardu; drsarducele@gmail.com

Received 21 April 2019; Accepted 21 April 2019; Published 19 May 2019

Copyright ( 2019 Celestino Sardu et al. This is an open access article distributed under the Creative Commons Attribution License, which permits unrestricted use, distribution, and reproduction in any medium, provided the original work is properly cited.

There are $\sim 415$ million people living with diabetes mellitus worldwide, with type 2 diabetes (T2DM) accounting for more than $90 \%$ of diabetic patients $[1,2]$. T2DM negatively affects the prognosis of patients by markedly increasing both hospitalization and mortality rate [1]. The common phenotype of T2DM is characterized by relative insulin deficiency caused by pancreatic $\beta$-cell dysfunction and insulin resistance in target organs [2-4]. These aspects eventually cause an altered glucose homeostasis, with consequent systemic negative effects on molecular and cellular functions [5-7]. Coming in the merit of the present editorial, we edited the Special Issue "Diabetes Mellitus and Its Cardiovascular Complications: New Insights into an Old Disease," collecting the state-of-the-art research in the field. Indeed, T2DM is a relevant cardiovascular $(\mathrm{CV})$ risk that is known to be the leading cause of morbidity and mortality associated with T2DM [8]. Insulin resistance and hyperglycemia work together as continuous negative triggers impairing ionic channel activity, the epigenetic program, and the cellular function of several organs [8]. At the clinical level, T2DM is strongly associated with both micro- and macrovascular complications, including retinopathy, nephropathy, and neuropathy, as well as cerebrovascular disease, ischemic heart disease (IHD), and peripheral artery disease (PAD) [1,9]. Several important concepts need to be highlighted: (1) diabetic nephropathy, cardiomyopathy, and PAD are frequently diagnosed at later disease stages; (2) screening programs are inconsistent and often inadequate to reduce the burden of these disorders [1]; (3) therefore, diet, exercise training, and lifestyle changes remain useful tools in preventing or at least delaying $\mathrm{CV}$ complications of T2DM $[1,10]$. Remarkably, dysfunctional ionic channels can be detected in T2DM patients without structural heart disease by direct alterations of ionic currents [11] as well as in patients with concomitant heart failure (HF) [12]. T2DM might increase the risk of atherosclerosis [1] alongside with a loss of regenerative myocardial muscle functions during an acute coronary syndrome. Intriguingly, T2DM might also cause functional alterations in the absence of obstructive coronary stenosis [13]. Indeed, altered glucose homeostasis and insulin resistance might trigger an advanced atherosclerosis in coronary arteries in cases with obstructive coronary stenosis and also in patients with nonobstructive coronary stenosis $[13,14]$. To date, T2DM has been shown to determine abnormalities in the dynamic responses to vasoactive stimuli, leading to increased rates of major adverse cardiac events (MACE) [13-15].On the other hand, T2DM might cause complex electrical alterations in $\mathrm{HF}$ patients increasing the risk of atrial and ventricular arrhythmias [16]. Specifically, T2DM induces alterations of ionic currents affecting action potential genesis and propagation in cardiac chambers, increasing automaticity and reentry mechanisms and favoring both atrial and ventricular arrhythmias [16]. Additionally, the increased inflammation and advanced cardiac fibrosis can lead to mechanical abnormalities of cardiac 
muscle with severe pump failure as well as higher rate of congestive HF and hospital admissions for HF worsening $[15,16]$. In this setting, new drug therapies such as interventional treatments have been developed to revert these negative conditions in order to ameliorate not only coronary and cardiac function but also clinical prognosis in IHD and HF patients with T2DM [14-16]. Moreover, there is an increasing necessity to develop new diagnostic tools for early detection of CV complications as well as new efficient treatments for this pathological condition. Thereby, a better understanding of specific diabetes genotypes and phenotypes might result in more specific and tailored management of T2DM patients [1]. Hence, there is an urgent need to find new therapeutic approaches to blunt the systemic and tissue-specific effects of hyperglycemia and insulin resistance and to reduce the development of diabetic CV complications. In conclusion, we believe that the main goal in the near future will be to find treatments better tailored to diabetic patients using a personalized-medicine approach.

\section{Conflicts of Interest}

The authors declare that there is no conflict of interest regarding the publication of this article.

Celestino Sardu
Claudio De Lucia
Markus Wallner
Gaetano Santulli

\section{References}

[1] S. Chatterjee, M. J. Davies, S. Heller, J. Speight, F. J. Snoek, and K. Khunti, "Diabetes structured self-management education programmes: a narrative review and current innovations," The Lancet Diabetes \& Endocrinology, vol. 6, no. 2, pp. 130$142,2018$.

[2] NCD Risk Factor Collaboration (NCD-RisC), "Worldwide trends in diabetes since 1980: a pooled analysis of 751 population-based studies with 4.4 million participants," The Lancet, vol. 387, no. 10027, pp. 1513-1530, 2016.

[3] A. Ortega, G. Berna, A. Rojas, F. Martin, and B. Soria, "Genediet interactions in type 2 diabetes: the chicken and egg debate," International Journal of Molecular Sciences, vol. 18, no. 6, p. 1188, 2017.

[4] A. Lombardi, J. Gambardella, X. L. Du et al., "Sirolimus induces depletion of intracellular calcium stores and mitochondrial dysfunction in pancreatic beta cells," Scientific Reports, vol. 7, no. 1, article 15823, 2017.

[5] P. Gæde, P. Vedel, N. Larsen, G. V. H. Jensen, H.-H. Parving, and O. Pedersen, "Multifactorial intervention and cardiovascular disease in patients with type 2 diabetes," The New England Journal of Medicine, vol. 348, no. 5, pp. 383-393, 2003.

[6] G. Santulli, "Childhood obesity and education," The Lancet Diabetes \& Endocrinology, vol. 4, no. 11, p. 957, 2016.

[7] J. Gambardella and G. Santulli, "Integrating diet and inflammation to calculate cardiovascular risk," Atherosclerosis, vol. 253, pp. 258-261, 2016.
[8] G. Santulli, G. Pagano, C. Sardu et al., "Calcium release channel RyR2 regulates insulin release and glucose homeostasis," The Journal of Clinical Investigation, vol. 125, no. 5, pp. 1968-1978, 2015.

[9] G. Santulli, "The adrenergic system in cardiovascular metabolism and aging," in The Cardiovascular Adrenergic System, A. Lymperopoulos, Ed., pp. 97-116, Springer, 2015.

[10] C. Sardu, G. Carreras, S. Katsanos et al., "Metabolic syndrome is associated with a poor outcome in patients affected by outflow tract premature ventricular contractions treated by catheter ablation," BMC Cardiovascular Disorders, vol. 14, no. 1, p. 176, 2014.

[11] R. Marfella, C. Sardu, P. Calabro et al., "Non-ST-elevation myocardial infarction outcomes in patients with type 2 diabetes with non-obstructive coronary artery stenosis: effects of incretin treatment," Diabetes, Obesity and Metabolism, vol. 20, no. 3, pp. 723-729, 2018.

[12] C. Sardu, P. Paolisso, C. Sacra et al., "Effects of metformin therapy on coronary endothelial dysfunction in prediabetic patients with stable angina and non obstructive coronary artery stenosis: the CODYCE multicenter prospective study," Diabetes Care, 2019.

[13] C. Sardu, P. Paolisso, C. Sacra et al., "Cardiac resynchronization therapy with a defibrillator (CRTd) in failing heart patients with type 2 diabetes mellitus and treated by glucagon-like peptide 1 receptor agonists (GLP-1 RA) therapy vs. conventional hypoglycemic drugs: arrhythmic burden, hospitalizations for heart failure, and CRTd responders rate," Cardiovascular Diabetology, vol. 17, no. 1, p. 137, 2018.

[14] C. Sardu, M. Barbieri, M. Santamaria et al., "Multipolar pacing by cardiac resynchronization therapy with a defibrillators treatment in type 2 diabetes mellitus failing heart patients: impact on responders rate, and clinical outcomes," Cardiovascular Diabetology, vol. 16, no. 1, p. 75, 2017.

[15] C. Sardu, M. Barbieri, M. L. Balestrieri et al., "Thrombus aspiration in hyperglycemic ST-elevation myocardial infarction (STEMI) patients: clinical outcomes at 1-year follow-up," Cardiovascular Diabetology, vol. 17, no. 1, p. 152, 2018.

[16] C. Sardu, N. D’Onofrio, C. Mauro, M. L. Balestrieri, and R. Marfella, "Thrombus aspiration in hyperglycemic patients with high inflammation levels in coronary thrombus," Journal of the American College of Cardiology, vol. 73, no. 4, pp. 530531, 2019. 


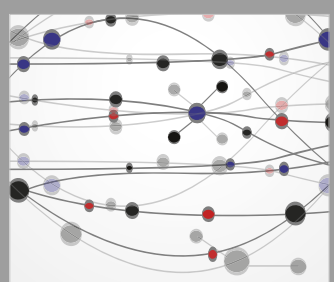

The Scientific World Journal
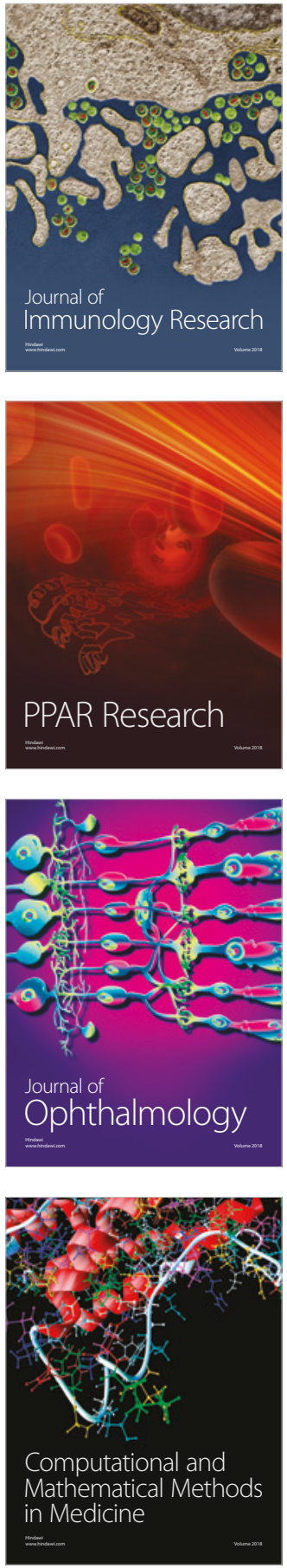

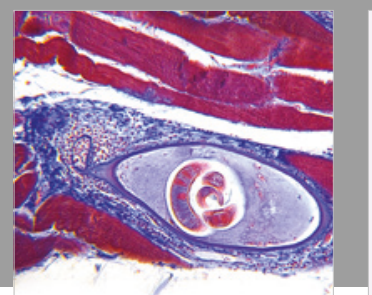

Gastroenterology Research and Practice

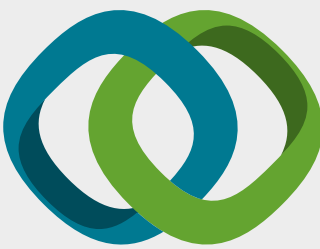

\section{Hindawi}

Submit your manuscripts at

www.hindawi.com
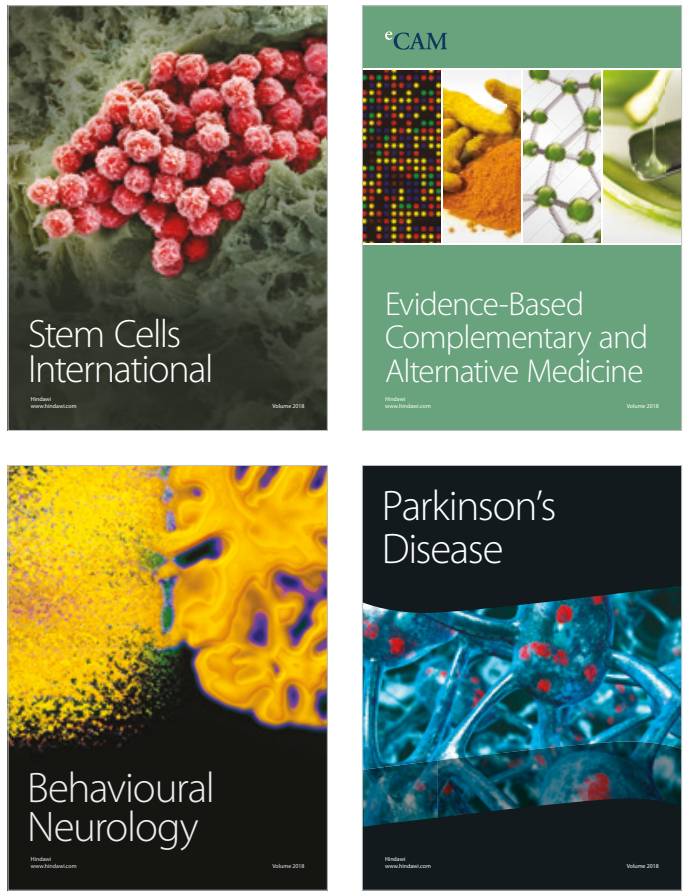

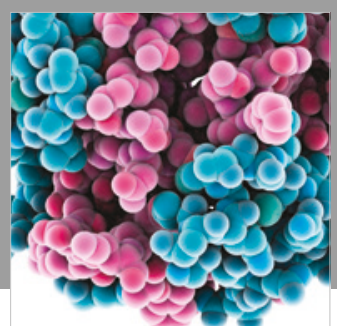

ournal of

Diabetes Research

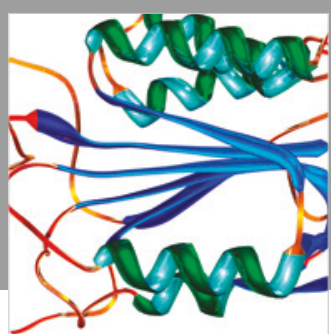

Disease Markers
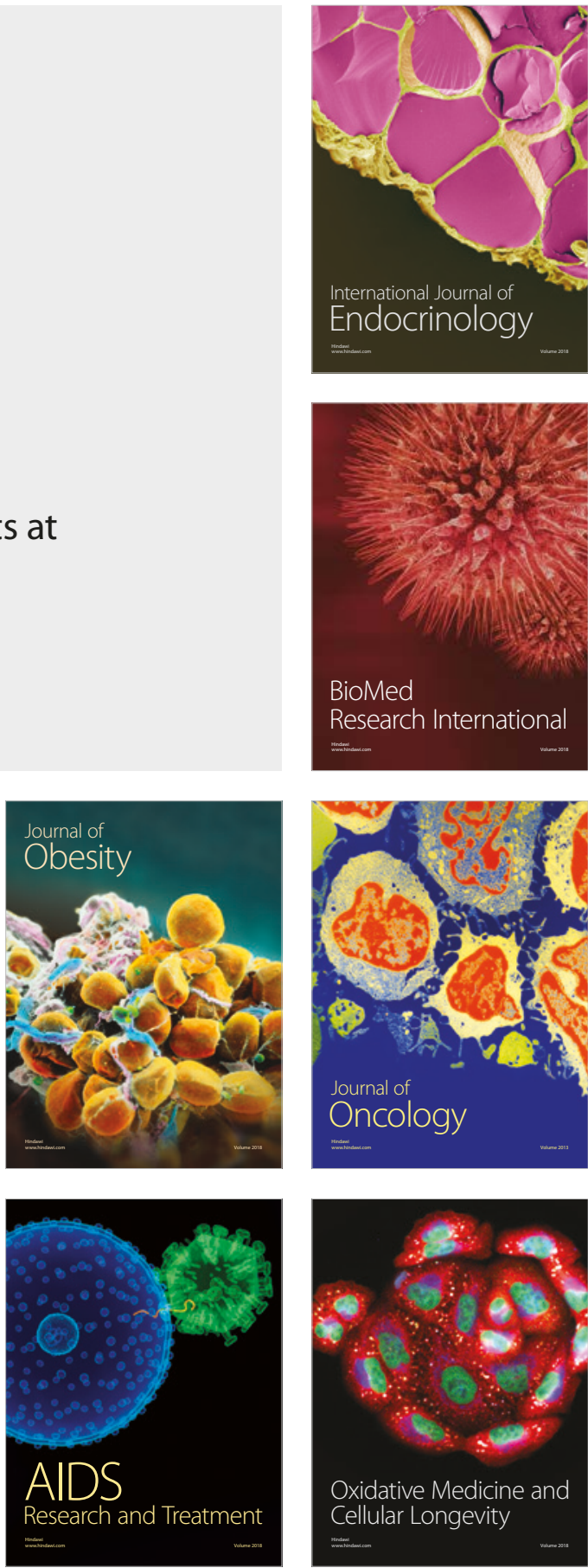